\title{
26 Research Square \\ Prevalence of Dry Eye Disease Among Chinese High School Students During the COVID-19 Outbreak
}

Feng Lin

Shanghai Jiaotong University School of Medicine

Yuying Cai

Shanghai Jiaotong University School of Medicine

Xinfeng Fei

Shanghai Fourth People's Hospital Affiliated to Tongji University School of Medicine

Yimin Wang

Shanghai Jiaotong University School of Medicine

Minwen Zhou

Shanghai Jiaotong University School of Medicine

Yan Liu ( $D$ liuyanliuyanxxx@163.com )

Shanghai Jiaotong University School of Medicine

\section{Research Article}

Keywords: dry eye disease, prevalence, high school, risk factors, COVID-19

Posted Date: February 21st, 2022

DOI: https://doi.org/10.21203/rs.3.rs-1360337/v1

License: (c) (i) This work is licensed under a Creative Commons Attribution 4.0 International License.

Read Full License

Version of Record: A version of this preprint was published at BMC Ophthalmology on April 26th, 2022. See the published version at https://doi.org/10.1186/s12886-022-02408-9. 


\section{Abstract}

\section{Purpose:}

The study aimed to investigate the prevalence of dry eye disease (DED) and relevant risk factors among Chinese high school students during the COVID-19 outbreak.

\section{Methods:}

A cross-sectional study was conducted from November to December 2020, and 4825 high school students from nine high schools in Shanghai were recruited. All students completed ocular surface disease index (OSDI) and perceived stress scale (PSS) questionnaires and answered other questions designed to ascertain information on the risk factors related to DED. DED was diagnosed when OSDI scores were greater than or equal to 13. The prevalence of symptomatic DED was determined. A T-test, Kruskal-Wallis test, Chi-square test, and logistic regression analysis were used to examine the possible risk factors.

\section{Results:}

The prevalence of symptomatic DED among Chinese high school students was $70.5 \%$. In univariate analysis, higher PSS scores $(P<0.001)$, prolonged video display terminal (VDT) use $(P<0.001)$, wearing contact lenses $(P=0.001)$, poor sleep quality $(P<0.001)$, and being female $(P<0.001)$ were significantly correlated with dry eyes. In multivariate logistic regression analysis, higher PSS scores $(P<0.001$, $\mathrm{OR}=1.20)$, prolonged VDT use $(P<0.001, \mathrm{OR}=1.07)$, poor sleep quality $(P<0.001, \mathrm{OR}=1.84)$, and being female $(P=0.001, \mathrm{OR}=1.25)$ were significant risk factors associated with $D E D$.

\section{Conclusions:}

Due to the epidemic, most Chinese high school students are in a high-risk environment in which they are more likely to suffer from DED, such as long online courses and heavy stress from school. Relevant preventive measures that may have a positive impact on public health and quality of life for high school students should be brought to the forefront.

\section{Introduction}

Dry eye disease (DED) is a condition occurring worldwide that is defined as a disorder of the tear film due to tear deficiency or excessive tear evaporation. ${ }^{[1]}$ It could cause damage to the interpalpebral ocular surface and is correlated with symptoms of ocular discomfort. ${ }^{[1]}$ Many epidemiological surveys regarding DED have been conducted around the world. ${ }^{[2]}$ However, most of these surveys involved relatively older populations, over 40 years of age, and few population-based DED studies focused on high school students. ${ }^{[2]}$ A population-based study investigating the prevalence of DED among Chinese college students revealed that the prevalence rate of DED reached $66.9 \% .{ }^{[3]}$ DED is particularly common in the 
young population in China. ${ }^{[3,4]}$ In addition, due to the coronavirus disease outbreak in 2019 (COVID-19), online courses have become much more prevalent among high school students. ${ }^{[5]}$ As excessive use of screens is one of the major courses of DED, students having long online courses may suffer from DED. ${ }^{[6}$,

7] Therefore, the prevalence of DED among high school students should not be neglected. It is necessary to conduct a preliminary survey on DED prevalence among Chinese high school students during the COVID-19 outbreak. In addition, considering the severe consequences of DED, such as keratitis, corneal ulcers, or even corneal perforation, early prevention of DED is important. By identifying significant risk factors, relevant preventive measures against DED could be implemented among high school students.

In this study, the prevalence and risk factors related to DED were analyzed among Chinese high school students who had taken online courses for at least half year at home and just returned to school.

\section{Methods}

\subsection{Sample Size Calculation}

A pre-survey was conducted in a randomly selected high school (323 subjects were involved). The prevalence rate of DED was $73.07 \%$. The formula $n=Z^{2} p(1-p) / \delta^{2}$ was used in the study (here, $a=0.05$, $Z=1.96, p=73.07 \%, \delta=0.02$ ). Additionally, a nonresponse rate of $5 \%$ was considered. Therefore, a minimum of 1990 subjects was required in this study.

\subsection{Sampling and Survey}

A cross-sectional survey was conducted among public high schools in Hongkou District in Shanghai from November to December 2020. Letters were sent to the principals of all 11 public high schools (according to the public high school list published by Hongkou District Education Bureau; http://www.shhk.gov.cn/hkjy/) located in Hongkou district in Shanghai. The letter explained the purpose of the study. Finally, nine public high schools consented to the study. All students in the nine schools were recruited. A total of 4825 subjects were included in our survey. Written informed consent was obtained from all subjects and parents of subjects of age group less than 16 . The study was approved by the Ethics Committee of Shanghai General Hospital and adhered to the tenets of the Declaration of Helsinki (NO. 2020KY026).

\subsection{OSDI Questionnaire}

The ocular surface disease index questionnaire (OSDI questionnaire) is one of the most widely used questionnaires to evaluate DED. ${ }^{[8,9]}$ It is viewed as one of the gold standards regarding DED according to the Dry Eye Work Shop (DEWS) consensus. ${ }^{[10]}$ In this study, a simplified Chinese version of the OSDI questionnaire, whose reliability and validity was previously verified, was utilized. ${ }^{[11]}$ It was divided into three dimensions: ocular symptoms, vision-related function, and environmental trigger scores. The ocular symptoms score consisted of three items, namely "eyes that are sensitive to light," "eyes that feel gritty," and "painful or sore eyes." The vision-related function score consisted of five items: "blurred vision," "poor 
vision," "reading," "driving at night," "working with a computer or bank machine," and "watching TV." The environmental triggers score consisted of three items: "windy conditions," "places or areas with low humidity," and "areas that are air conditioned." The severity was graded on a scale of 0 to 5 according to the frequency regarding each item, which represented "none of the time," "some of the time," "half of the time," "most of the time," and "all the time." The OSDI score was based on the formula: [(sum of scores for all questions answered) *100] / [(total number of answered questions)*4]. Using the formula, the total OSDI score could be calculated.

\subsection{Definition of DED}

According to the total OSDI score, DED was considered as "symptomatic DED" and was defined as a subject whose OSDI score was greater than or equal to $13^{[12,13]}$. Additionally, subjects were classified into 4 categories by OSDI score: normal (scores $0-12$ ), mild DED (13-22), moderate DED (23-32), and severe DED $(33-100)^{[12]}$.

\subsection{Definition of risk factors}

In total, five questions were related to potential risk factors, namely "psychological stress," "VDT use," "wearing contact lenses," "sleep quality," and "gender." To measure and evaluate psychological stress, the Chinese version of the perceived stress scale (PSS) was utilized. The PSS questionnaire is one of the most widely used psychological instruments for measuring the perception of stress. Developed by Cohen in 1983 , it has shown sufficient reliability and validity. ${ }^{[14]}$ The Chinese version of the PSS questionnaire was also found to be reliable. ${ }^{[15,16]}$ Originally, it consisted of 14 items that were designed to determine how unpredictable, uncontrollable, and overloaded respondents find their lives. To facilitate the implementation, the four items version of PSS was adapted for this study. This version consisted of four PSS items, two of which were negative ("how often have you felt that you were unable to control the important things in your life" and "how often have you felt difficulties were piling up so high that you could not overcome them"), while the other two items were positive ("how often have you felt confident about your ability to handle your personal problems" and "how often have you felt that things were going your way"). The subjects were required to answer each question using a five-point Likert scale score ranging from 0 (never) to 4 (very often) and report the event frequency correlated with the PSS items in the last month. PSS scores were obtained by reversing responses $(0=4,1=3,2=2,3=1,4=0)$ to the two positively stated items and summing all scale items. The total scores ranged from 0 to 16 , and subjects with higher scores had higher psychological stress levels. The video display terminal (VDT) use question was defined as "the average hours spent on VDT devices, such as iPad, iPhone, or computers, per day for the last week." The wearing contact lenses question was defined as wearing a contact lens at least once a week for the last three months. Poor sleep quality was defined as difficulty in falling asleep.

\subsection{Research process}

Before the formal survey began, the project leader trained project members and standardized the survey process. On the day of the survey, the subject had to fill out a questionnaire that consisted of four parts 
(Supplemental Figure 1):

(1) General information: name, gender, age, school, class, and student identification number

(2) OSDI questionnaire

(3) DED risk factors (contact lens wear, sleep quality and VDT use) and medical history regarding eye diseases (self-reported ocular inflammation, such as conjunctivitis and keratitis, or previous ocular surgery history within the last 6 months)

(4) PSS questionnaire

Questionnaires with omissions were considered invalid. Two project members transferred the data to a database independently. Subjects who had a history of any ocular inflammation or ocular surgery within the last six months were excluded from the survey.

\subsection{Statistical Analyses}

The normality of the data distribution was tested using the Kolmogorov-Smirnov test. Normal distribution parameters were expressed as the mean (standard deviation, SD). Non-normal distribution parameters were expressed as the median (interquartile range, IQR). The prevalence of DED was calculated as a ratio. In univariate analysis, normally distributed continuous variables were compared by a T-test, while non-normally distributed continuous variables were compared by the Mann-Whitney U test. Chi-square tests were used for comparisons between categorical variables. All risk factors were then introduced into a binary logistic regression model. Relative risks were estimated as odds ratios (OR) with a $95 \%$ confidence interval $(\mathrm{Cl}) . P<0.05$ was regarded as a significant difference. Data analyses were carried out using SPSS version 21.0 (SPSS, Chicago, IL, USA).

\section{Results}

In this study, a total of 4722 valid questionnaires were included. The questionnaire reclaiming efficiency was $97.9 \%$. Among the 4722 subjects in our selected schools, 28 subjects were excluded because of ocular inflammation or ocular surgery. A flowchart pertaining to the study is shown in Supplemental Fig. 2.

In total, 4694 subjects were included in our survey, including 2248 males and 2446 females. The mean age was 16.39 (0.95), which ranged from 14 to 19. Detailed characteristics regarding the subjects are summarized in Table 1.

Symptomatic DED was present in 3311 subjects. The prevalence of symptomatic DED was $70.5 \%$. According to the OSDI scores, all DED subjects were then divided into three groups, in which 1154 subjects reported mild ocular surface symptoms (13-22), 1063 subjects reported moderate ocular 
surface symptoms (23-32), and 1094 subjects reported severe ocular surface symptoms (33-100). The distribution of OSDI scores is presented in Fig. 1.

Among the 12 items from the OSDI questionnaire in 3311 DED subjects, the most frequently (item score $\geq 1$ ) reported ocular symptom was painful or sore eyes (89.3\%). The most frequently (item score $\geq 1$ ) reported vision-related function problem was blurred vision ( $81.1 \%)$. The most frequently (item score $\geq 1$ ) reported environmental trigger was windy conditions (72.3\%). The distribution of the scores in the 12 items from the OSDI questionnaire is presented in Fig. 2.

In the univariate analysis, PSS scores were higher in DED subjects than in normal subjects $(P<0.001)$. Additionally, the DED group had a longer VDT use time $(P<0.001)$ (Supplemental Table 1). Wearing contact lenses $(P=0.012)$, poor sleep quality $(P<0.001)$, and being female $(P<0.001)$ were significantly correlated with DED (Supplemental Table 2).

Table 2 shows the binary logistic regression model. DED subjects had higher PSS scores $(O R=1.20, P<$ $0.001)$ and spent more time on VDT devices $(O R=1.07, P<0.001)$. Poor sleep quality $(O R=1.84, P<$ $0.001)$ and being female $(\mathrm{OR}=1.25, P=0.001)$ were still risk factors related to $\mathrm{DED}$. Although wearing contact lenses was related to DED in univariate analysis, it was not a risk factor significantly related to DED in the binary logistic regression analysis $(P=0.152)$.

\section{Discussion}

As the first epidemiological survey on high school students since the outbreak of COVID-19 around the world, the prevalence of symptomatic DED among Chinese high school students was $70.5 \%$. In addition, the percentages of students in the mild (24.6\%), moderate (22.6\%), and severe DED groups (23.3\%) were similar. The results were further compared with other DED epidemiological surveys that also adopted the same DED diagnostic criteria based on OSDI questionnaires. ${ }^{[3]}$ Han et al. ${ }^{[3]}$ conducted a survey on the prevalence of DED among Chinese college students in Guangzhou in 2016 and showed that the prevalence of symptomatic DED was $66.9 \%$, of which the subjects with mild, moderate, and severe DED accounted for $27.3,16.4$, and $16.7 \%$, respectively. In contrast, the prevalence of DED among high school students in this study was higher, and the proportion of moderate and severe DED subjects was also higher than that found in Han et al. ${ }^{[3]}$ As for other epidemiological surveys on DED among high school students, Zhang et al. ${ }^{[4]}$ conducted an epidemiological survey amongst Chinese high school students in 2010 that revealed that the prevalence of symptomatic DED was $23.7 \%$. In contrast, Uchino et al. ${ }^{[17]}$ reported that the prevalence of symptomatic DED among Japanese high school students was $21.6 \%$ in 2008. Both studies adopted the Schaumberg questionnaire ${ }^{[18]}$ as the DED diagnostic tool and showed similar prevalence rates of DED among high school students. As the prevalence rate they reported was far lower than that in this study, it may be attributed to different DED diagnostic tools.

Among all OSDI questionnaire items, blurred vision was the most frequently reported vision-related function problem by DED subjects. This could be due to ocular surface damage in the overlying optical 
zone, which could cause higher-order aberrations and increased corneal backward light scattering. ${ }^{[19]}$ Additionally, it was difficult to detect visual or optical changes using standard visual acuity testing. As student vision screening is conducted annually in high schools, some students may complain about blurred vision though their vision acuity is good. This may imply the progress of DED. Early screening tests for DED and preventive measures should be noted.

The PSS questionnaire is one of the most widely used psychological instruments for measuring the perception of stress. As the PSS questionnaire has already been used to evaluate psychological stress among senior students ${ }^{[16]}$, it was the first time that we evaluated the correlation between psychological stress and DED in this population. Higher psychological stress could lead to DED and could cause anxiety. As the correlation of anxiety with DED has been demonstrated in several studies ${ }^{[20,21]}$, psychological stress may induce DED. In anxiety subjects, disturbances of serotonin receptors, located around the conjunctival epithelium, could influence Meibomian glands, which can lead to tear film deficiency. ${ }^{[20,22]}$ However, with the pain and discomfort that DED subjects may experience, it could inversely contribute to anxiety. ${ }^{[23]}$ Finally, these factors composed a vicious circle that led to DED. In addition, the high PSS scores among Chinese high school students (median score in DED subjects: 8.00, in non-DED subjects: 6.00 ), which were comparable to those of medical students, should be highlighted. ${ }^{[24]}$ This may be attributed to the fact that students under East Asian culture are more likely to show higher depression and lower life satisfaction than their European counterparts due to the emphasis on academic performance. ${ }^{[25]}$

Prolonged VDT use has been viewed as a risk factor related to DED in many studies ${ }^{[26,27]}$, which was corroborated in this study. Cumulative years of VDT use may change lacrimal gland function and ultimately result in decreased tear secretion. ${ }^{[28]}$. Furthermore, prolonged VDT users have shorter tear film break-up time ${ }^{[26]}$, which may contribute to prolonged blinking intervals while gazing at VDT devices. ${ }^{[29]} \mathrm{A}$ diminished blink frequency rate and incomplete blinking could also contribute to DED. ${ }^{[29]}$ As the use of iPads, iPhones, and laptops is much more common than before, especially in young teenagers ${ }^{[30]}$, the high risk of prolonged VDT use causing DED should be highlighted.

As a risk factor related to DED in this study, several studies have demonstrated a correlation between sleep quality and DED. ${ }^{[31-33]}$ Sleep deprivation could disrupt the lacrimal system and induce DED. ${ }^{[32]}$ With poor sleep quality, parasympathetic tone can be reduced, as removal of parasympathetic innervation to the lacrimal gland can lead to a rapid reduction in tear flow and induced DED. ${ }^{[33]}$ Additionally, a high proportion of poor sleep quality (32.53\%) among all high school students was noted in our study. This could be due to the fact that Chinese high school students often have a heavy workload and fierce competition for university entrance and parents' high expectations of their academic achievement. ${ }^{[34]}$

Females showed a 1.25-fold higher risk of having DED than males. Being female has been identified as a significant risk factor for the development of DED in many studies ${ }^{[35,36]}$, which could largely be attributed to the effects of sex steroids, such as androgens and estrogens. Females have unique hormonal cycles 
(menstruation, pregnancy, and menopause) that can affect tear film stability. For example, a study showed that a low androgen level was a consistent risk factor for DED. ${ }^{[36]}$ Although the association of sex steroids with DED has not been fully determined, their influence should be considered.

Concerning the significantly correlated risk factors found in this study, taking precautions is necessary to reduce the prevalence of DED. For example, students' mental status should be taken seriously, and school staff should appropriately alleviate students' academic pressure. Consolation or even psychological counseling performed by school doctors for students who have poor sleep quality and heavy stress is recommended. In addition, students should be persuaded to reduce VDT use time. A one-minute break for every 30 minutes of VDT use or deliberately excessive blinking is highly recommended.

Wearing contact lenses has been viewed as a risk factor related to DED in many studies. ${ }^{[17,37], \text { When a }}$ contact lens is placed on the ocular surface, the tear film becomes separated into the pre- and post-lens tear film. Because the tear film becomes separated, the volume of the aqueous layer at the pre-lens tear film is decreased and becomes unstable and easily breakable within a short period after blinking. ${ }^{[38]}$ However, it was not a significant risk factor related to DED in this study. This might be due to the different proportions of subjects wearing contact lenses. Compared with an epidemiological survey among Japanese high school students, $37.8 \%$ of students wore contact lenses, while only $9.71 \%$ of students used contact lenses in this study.

This study has some limitations and biases. First, only nine high schools in Shanghai were included in our survey. Schools in other areas in China were not covered in this study. Although this does not rule out selection bias, it is still the largest population-based epidemiological survey among Chinese high school students. This study had a large sample size as it was well over that recommended by statistical tests. Second, other risk factors related to DED, such as diabetes and smoking, were not analyzed in the study. [39, 40] However, the prevalence rate of diabetes among high school students is pretty low, and smoking is forbidden in Chinese high schools. Finally, our study lacked the parameters of dry eye signs, and the prevalence of DED could vary from different diagnostic criteria. Only the prevalence of symptomatic DED based on the OSDI questionnaire was reported in this study. Further study should incorporate objective dry eye examinations to further validate the results from the symptom questionnaires.

\section{Conclusion}

In summary, this cross-sectional study demonstrated a fairly high prevalence rate of DED among Chinese high school students during the COVID-19 outbreak for the first time. In addition, there were some significant risk factors, such as increased psychological stress, prolonged VDT use, poor sleep quality, and being female. As students were affected by the pandemic, most Chinese high school students are in a high-risk environment in which they may suffer from DED, such as long online courses and heavy stress from school. Relevant preventive measures that may have a positive impact on public health and quality of life for high school students should be brought to the forefront. 


\section{Declarations}

-Ethics approval and consent to participants: Written informed consent was obtained from all subjects and parents of subjects of age group less than 16. The study was approved by the Ethics Committee of Shanghai General Hospital and adhered to the tenets of the Declaration of Helsinki (NO. 2020KY026).

-Consent to publish: not applicable.

-Availability of data and materials: Dataset analyzed in the current study are available in the supplemental materials which is a spreadsheet named "Data".

-Competing interests: The authors report no conflicts of interest and have no proprietary interest in any of the materials mentioned in this article.

-Funding: The study was supported by the Shanghai Natural Science Foundation (19ZR1440900), Shanghai Pujiang Program (2019PJD047).

-Authors' Contributions: Authors contributions: All authors read and approved the final manuscript. Research concept and design (YL); data collection of the research (FL, YYC, XFF, YMW, MWZ); analysis and interpretation of data (FL); writing of the manuscript (FL, YYC, XFF).

-Acknowledgements: Not applicable.

\section{References}

1. Craig JP, Nichols KK, Akpek EK, Caffery B, Dua HS, Joo C-K, Liu Z, Nelson JD, Nichols JJ, Tsubota K et al. TFOS DEWS II Definition and Classification Report. Ocul Surf. 2017; 15(3):276283.doi:10.1016/j.jtos.2017.05.008

2. Stapleton F, Alves M, Bunya VY, Jalbert I, Lekhanont K, Malet F, Na K-S, Schaumberg D, Uchino M, Vehof J et al. TFOS DEWS II Epidemiology Report. Ocul Surf. 2017; 15(3):334365.doi:10.1016/j.jtos.2017.05.003

3. Han L, Luo X, Zhou YM, Liu XL, Chen QS. Prevalence and influencing factors of dry eyes syndrome among college students in Guangzhou City. Occup and Health 2016; 32(21):29882990.doi:10.13329/j.cnki.zyyjk.2016.0940

4. Zhang Y, Chen H, Wu X. Prevalence and Risk Factors Associated with Dry Eye Syndrome among Senior High School Students in a County of Shandong Province, China. Ophthalmic Epidemiology. 2012; 19(4):226-230.doi:10.3109/09286586.2012.670742

5. Li H, Liu S-M, Yu X-H, Tang S-L, Tang C-K. Coronavirus disease 2019 (COVID-19): current status and future perspectives. Int J Antimicrob Agents. 2020; 55(5):105951.doi:10.1016/j.jijantimicag.2020.105951

6. Carbonell X, Chamarro A, Oberst U, Rodrigo B, Prades M. Problematic Use of the Internet and Smartphones in University Students: 2006-2017. Int J Environ Res Public Health. 2018; 
15(3).doi:10.3390/ijerph15030475

7. Condori-Meza IB, Dávila-Cabanillas LA, Challapa-Mamani MR, Pinedo-Soria A, Torres RR, Yalle J, Rojas-Humpire R, Huancahuire-Vega S. Problematic Internet Use Associated with Symptomatic Dry Eye Disease in Medical Students from Peru. Clin Ophthalmol. 2021; 15:43574365.doi:10.2147/opth.S334156

8. Sherry A, Aridi M, Ghach W. Prevalence and risk factors of symptomatic dry eye disease in Lebanon. Contact lens \& anterior eye : the journal of the British Contact Lens Association. 2020; 43(4):355358.doi:10.1016/j.clae.2019.08.001

9. Asiedu K, Kyei S, Boampong F, Ocansey S. Symptomatic Dry Eye and Its Associated Factors: A Study of University Undergraduate Students in Ghana. Eye Contact Lens. 2017; 43(4):262266.doi:10.1097/ICL.0000000000000256

10. Wolffsohn JS, Arita R, Chalmers R, Djalilian A, Dogru M, Dumbleton K, Gupta PK, Karpecki P, Lazreg S, Pult H et al. TFOS DEWS II Diagnostic Methodology report. Ocul Surf. 2017; 15(3):539-

574.doi:10.1016/j.jtos.2017.05.001

11. Zhao H, Liu Z, Yang W, Xiao X, Chen J, Li Q, Zhong T. Development and assessment of a dry eye questionnaire applicable to the Chinese population. Chinese Journal of Ophthalmology. 2015; 51(9):647-654.doi:10.3760/cma.j.issn.0412-4081.2015.09.003

12. Schiffman RM, Christianson MD, Jacobsen G, Hirsch JD, Reis BL. Reliability and validity of the Ocular Surface Disease Index. Arch Ophthalmol. 2000; 118(5):615-621.doi: 10.1001/archopht.118.5.615

13. Kobia-Acquah E, Ankamah-Lomotey S, Owusu E, Forfoe S, Bannor J, Koomson JA, Opoku M, Dzikpo DM, Mensah DNO, Amonoo JN et al. Prevalence and associated risk factors of symptomatic dry eye in Ghana: A cross-sectional population-based study. Contact lens \& anterior eye : the journal of the British Contact Lens Association. 2021:101404.doi:10.1016/j.clae.2020.12.067

14. Cohen S, Kamarck T, Mermelstein R. A global measure of perceived stress. J Health Soc Behav. 1983; 24(4):385-396.

15. Lu W, Bian Q, Wang W, Wu X, Wang Z, Zhao M. Chinese version of the Perceived Stress Scale-10: A psychometric study in Chinese university students. PLoS One. 2017; 12(12):e0189543e0189543.doi:10.1371/journal.pone.0189543

16. Liu X, Zhao Y, Li J, Dai J, Wang X, Wang S. Factor Structure of the 10-Item Perceived Stress Scale and Measurement Invariance Across Genders Among Chinese Adolescents. Front Psychol. 2020; 11:537537.doi:10.3389/fpsyg.2020.00537

17. Uchino M, Dogru M, Uchino Y, Fukagawa K, Shimmura S, Takebayashi T, Schaumberg DA, Tsubota K. Japan Ministry of Health Study on Prevalence of Dry Eye Disease Among Japanese High School Students. American Journal of Ophthalmology. 2008; 146(6):925-

929.e922.doi:10.1016/j.ajo.2008.06.030

18. Gulati A, Sullivan R, Buring JE, Sullivan DA, Dana R, Schaumberg DA. Validation and repeatability of a short questionnaire for dry eye syndrome. American journal of ophthalmology. 2006; 142(1):125- 
131.doi: 10.1016/j.ajo.2006.02.038

19. Koh S. Mechanisms of Visual Disturbance in Dry Eye. Cornea. 2016; 35 Suppl 1:S83-S88.doi: 10.1097/ICO.0000000000000998

20. Tiskaoglu NS, Yazıcı A, Karlıdere T, Sari E, Oguz EY, Musaoglu M, Aslan S, Samet Ermiş S. Dry Eye Disease in Patients with Newly Diagnosed Depressive Disorder. Current eye research. 2017; 42(5):672-676.doi:10.1080/02713683.2016.1236966

21. Ulusoy MO, Işık-Ulusoy S, Kıvanç SA. Evaluation of dry eye disease in newly diagnosed anxiety and depression patients using anterior segment optical coherence tomography. Eye and vision (London, England). 2019; 6:25.doi:10.1186/s40662-019-0149-y

22. Chhadva P, Lee T, Sarantopoulos CD, Hackam AS, McClellan AL, Felix ER, Levitt RC, Galor A. Human Tear Serotonin Levels Correlate with Symptoms and Signs of Dry Eye. Ophthalmology. 2015; 122(8):1675-1680.doi:10.1016/j.ophtha.2015.04.010

23. Castillo RC, Wegener ST, Heins SE, Haythornthwaite JA, Mackenzie EJ, Bosse MJ. Longitudinal relationships between anxiety, depression, and pain: results from a two-year cohort study of lower extremity trauma patients. Pain. 2013; 154(12):2860-2866.doi:10.1016/j.pain.2013.08.025

24. Hyon JY, Yang HK, Han SB. Dry Eye Symptoms May Have Association With Psychological Stress in Medical Students. Eye Contact Lens. 2019; 45(5):310-314.doi:10.1097/icl.0000000000000567

25. Stankov L. Depression and life satisfaction among European and Confucian adolescents. Psychological assessment. 2013; 25(4):1220-1234.doi:10.1037/a0033794

26. Uchino M, Yokoi N, Uchino Y, Dogru M, Kawashima M, Komuro A, Sonomura Y, Kato H, Kinoshita S, Schaumberg DA et al. Prevalence of dry eye disease and its risk factors in visual display terminal users: the Osaka study. American journal of ophthalmology. 2013; 156(4):759-

766.doi:10.1016/j.ajo.2013.05.040

27. Cheng X, Song M, Kong J, Fang X, Ji Y, Zhang M, Wang H. Influence of Prolonged Visual Display Terminal Use and Exercise on Physical and Mental Conditions of Internet Staff in Hangzhou, China. Int J Environ Res Public Health. 2019; 16(10).doi:10.3390/ijerph16101829

28. Nakamura S, Kinoshita S, Yokoi N, Ogawa Y, Shibuya M, Nakashima H, Hisamura R, Imada T, Imagawa $\mathrm{T}$, Uehara $\mathrm{M}$ et al. Lacrimal hypofunction as a new mechanism of dry eye in visual display terminal users. PLoS One. 2010; 5(6):e11119.doi:10.1371/journal.pone.0011119

29. Argilés M, Cardona G, Pérez-Cabré E, Rodríguez M. Blink Rate and Incomplete Blinks in Six Different Controlled Hard-Copy and Electronic Reading Conditions. Invest Ophthalmol Vis Sci. 2015; 56(11):6679-6685.doi:10.1167/iovs.15-16967

30. Marshall SJ, Gorely T, Biddle SJH. A descriptive epidemiology of screen-based media use in youth: a review and critique. J Adolesc. 2006; 29(3):333-349.doi: 10.1016/j.adolescence.2005.08.016

31. Wu M, Liu X, Han J, Shao T, Wang Y. Association Between Sleep Quality, Mood Status, and Ocular Surface Characteristics in Patients With Dry Eye Disease. Cornea. 2019; 38(3):311-

317.doi:10.1097/ICO.0000000000001854

Page $11 / 16$ 
32. Li S, Ning K, Zhou J, Guo Y, Zhang H, Zhu Y, Zhang L, Jia C, Chen Y, Sol Reinach P et al. Sleep deprivation disrupts the lacrimal system and induces dry eye disease. Exp Mol Med. 2018; 50(3):e451.doi:10.1038/emm.2017.285

33. Nguyen $\mathrm{DH}$, Vadlamudi $\mathrm{V}$, Toshida $\mathrm{H}$, Beuerman RW. Loss of parasympathetic innervation leads to sustained expression of pro-inflammatory genes in the rat lacrimal gland. Auton Neurosci. 2006; 124(1-2):81-89.doi: 10.1016/j.autneu.2005.12.001

34. Chan Y, Chan YY, Cheng SL, Chow MY, Tsang YW, Lee C, Lin CY. Investigating quality of life and selfstigma in Hong Kong children with specific learning disabilities. Research in developmental disabilities. 2017; 68:131-139.doi:10.1016/j.ridd.2017.07.014

35. Matossian C, McDonald M, Donaldson KE, Nichols KK, Maclver S, Gupta PK. Dry Eye Disease: Consideration for Women's Health. Journal of women's health (2002). 2019; 28(4):502514.doi:10.1089/jwh.2018.7041

36. Sriprasert I, Warren DW, Mircheff AK, Stanczyk FZ. Dry eye in postmenopausal women: a hormonal disorder. Menopause (New York, NY). 2016; 23(3):343-351.doi:10.1097/gme.0000000000000530

37. Uchino M, Nishiwaki Y, Michikawa T, Shirakawa K, Kuwahara E, Yamada M, Dogru M, Schaumberg DA, Kawakita T, Takebayashi T et al. Prevalence and risk factors of dry eye disease in Japan: Koumi study. Ophthalmology. 2011; 118(12):2361-2367.doi:10.1016/j.ophtha.2011.05.029

38. Kojima T. Contact Lens-Associated Dry Eye Disease: Recent Advances Worldwide and in Japan. Invest Ophthalmo/ Vis Sci. 2018; 59(14):Des102-des108.doi:10.1167/iovs.17-23685

39. Sandra Johanna GP, Antonio LA, Andrés GS. Correlation between type 2 diabetes, dry eye and Meibomian glands dysfunction. Journal of optometry. 2019; 12(4):256262.doi:10.1016/j.optom.2019.02.003

40. Makrynioti D, Zagoriti Z, Koutsojannis C, Morgan PB, Lagoumintzis G. Ocular conditions and dry eye due to traditional and new forms of smoking: A review. Contact lens \& anterior eye : the journal of the British Contact Lens Association. 2020; 43(3):277-284.doi:10.1016/j.clae.2020.02.009

\section{Tables}

Table 1. Subjects' characteristics 


\begin{tabular}{|ll|}
\hline High school students & $\mathrm{n}(\%)$ \\
\hline \multicolumn{1}{|c|}{ Male } \\
\hline Female & $2248(47.9)$ \\
\hline Age & $2446(52.1)$ \\
\hline 14 & $12(0.3)$ \\
\hline 15 & $870(18.5)$ \\
\hline 16 & $1720(36.6)$ \\
\hline 17 & $1482(31.6)$ \\
\hline 18 & $585(12.5)$ \\
\hline 19 & $25(0.5)$ \\
\hline Grade & $1662(35.4)$ \\
\hline Grade10 & $1568(33.4)$ \\
\hline Grade11 & $1464(31.2)$ \\
\hline Grade 12 & \\
\hline
\end{tabular}

Table 2. Binary logistic regression analysis of risk factors for DED 


\begin{tabular}{|lll|}
\hline Risk Factors & OR $(95 \% \mathrm{Cl})$ & $\mathrm{P}$ \\
\hline PSS score & $1.20(1.17-1.24)$ & $P<0.001$ \\
\hline VDT use time & $1.07(1.03-1.10)$ & $P<0.001$ \\
\hline Contact lens wear & & \\
\hline Yes & $1.19(0.94-1.50)$ & $P=0.152$ \\
\hline No & 1.00 (Ref) & \\
\hline Poor sleep quality & & \\
\hline Yes & $1.84(1.57-2.16)$ & $P<0.001$ \\
\hline No & $1.00($ Ref $)$ & \\
\hline Gender & & \\
\hline Female & $1.25(1.09-1.43)$ & $P=0.001$ \\
\hline Male & 1.00 (Ref) & \\
\hline
\end{tabular}

Figures

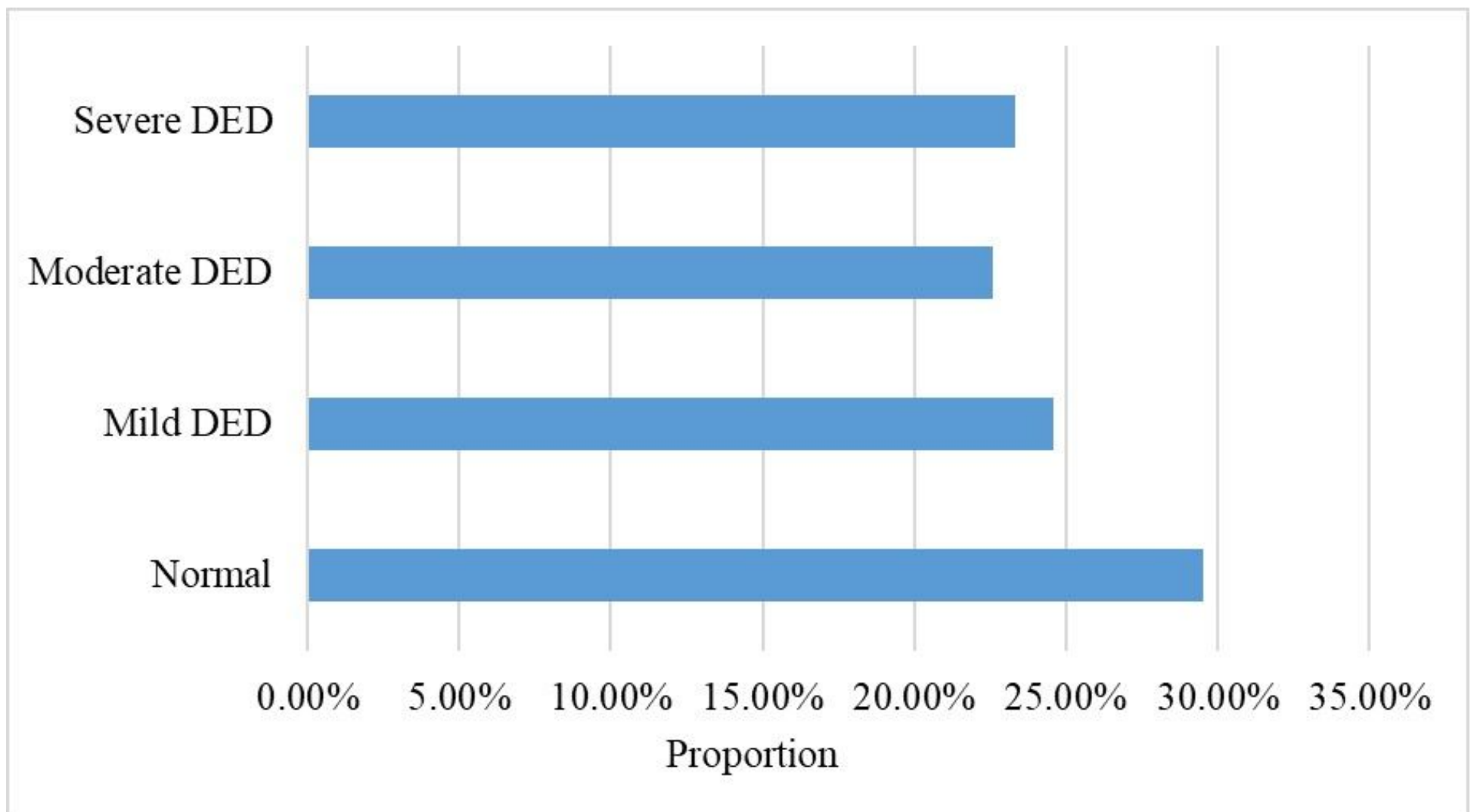

Figure 1 


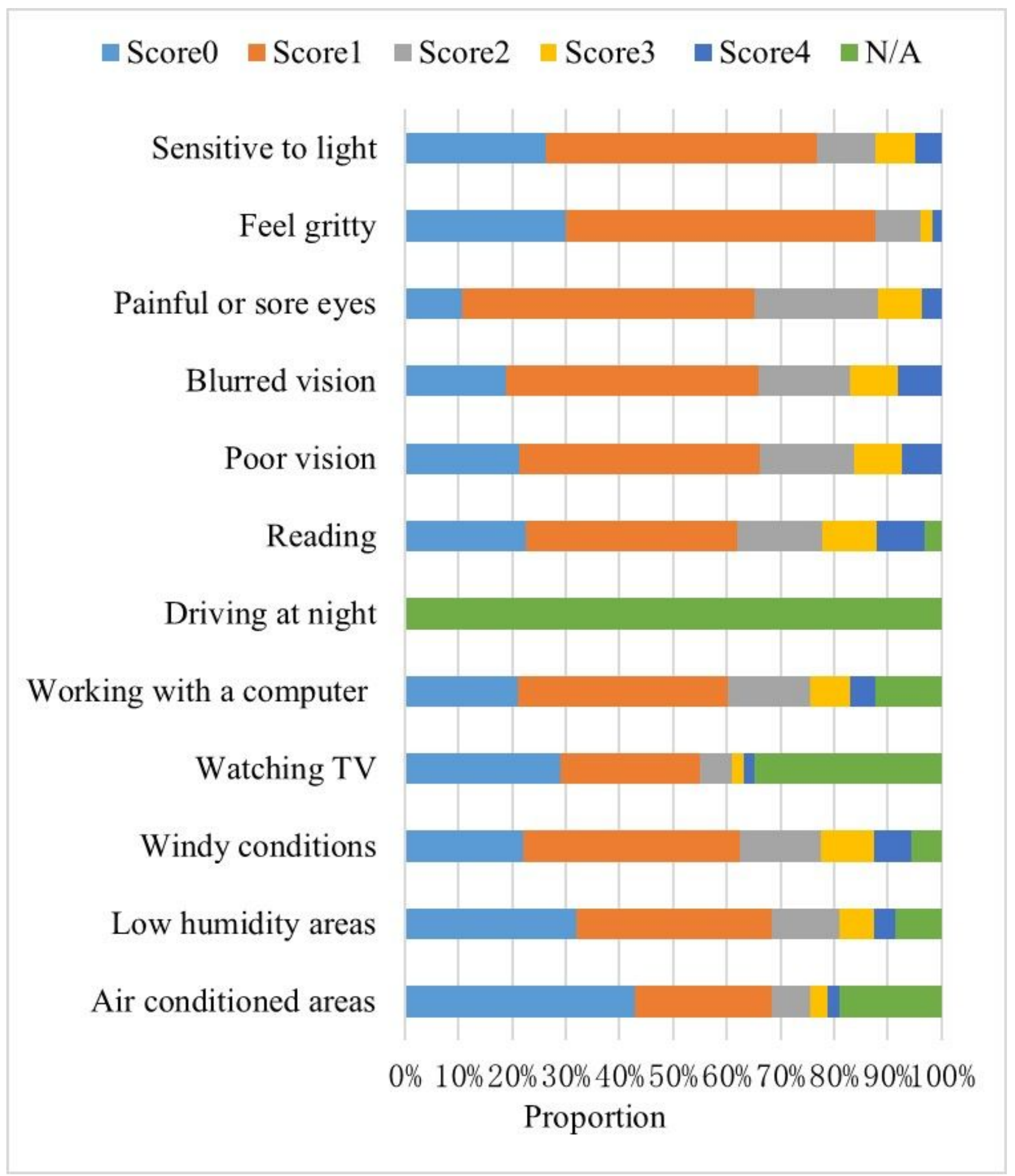

\section{Figure 2}

OSDI score by item in DED subjects Each question ranged from 0 to 4 according to the frequency ( $0=$ none of the time, $1=$ some of the time, $2=$ half of the time, $3=$ most of the time, $4=$ =all of the time for the last week). N/A meant the individual did not perform the task or stay in the situation for the last week. 


\section{Supplementary Files}

This is a list of supplementary files associated with this preprint. Click to download.

- Data.xlsx

- SupplementalFigure1.Researchquestionnaire.pdf

- SupplementalFigure2.Workflowofthestudy.pdf

- SupplementalTable1.UnivariateanalysisofriskfactorsforDEDcontinuousvariables.docx

- SupplementalTable2.UnivariateanalysisofriskfactorsforDEDcategoricalvariables.docx 